Divisiones de 'Pediatría, ${ }^{2}$ Salud Pública, ${ }^{3}$ Cardiología, y Ginecología. Facultad de Medicina, Pontificia Universidad Católica de Chile, Santiago, Chile. aDoctor en estadísticas. ${ }^{b}$ Enfermera universitaria. ¿Licenciada en estadísticas.

${ }^{d}$ Magíster en nutrición eTesista Magíster de nutrición. Proyecto FONDECYT regular 1090594

No hubo influencia de la agencia financiadora en el diseño de este estudio, ni en la recolección y análisis de los datos; tampoco en la preparación, revisión o aprobación del manuscrito.

Recibido el 28 de diciembre de 2011, aceptado el 30 de mayo de 2012.

Correspondencia a: Dr. Francisco Mardones Santander

Marcoleta 434, Santiago. Código Postal: 833-0073

Teléfono: 3546898.

E mail:mardones@med. puc.clofmardons@uc.c
${ }^{4}$ Nutrición y ${ }^{5}$ Obstetricia

Trabajo Financiado por

\section{La presión arterial es un importante marcador de ateroesclerosis subclínica en niños}

\author{
PILAR ARNAIZ ${ }^{1}$, LUIS VILLARROEL ${ }^{2, a}$, SALESA BARJA ${ }^{1}$, \\ IVÁN GODOY ${ }^{3}$, BERTA CASSIS ${ }^{1, \mathrm{~b}}$, ANGÉLICA DOMÍNGUEZ ${ }^{1, \mathrm{c}}$, \\ OSCAR CASTILLO ${ }^{4, d}$, MARCELO FARÍAS ${ }^{5}$, JACQUELINE CARVAJAL ${ }^{3}$, \\ ANA CRISTINA TINOCO ${ }^{4, e}$, FRANCISCO MARDONES ${ }^{2}$
}

\section{Association of carotid intima media thickness with blood pressure and HDL cholesterol in children}

Background: Cardiovascular risk factors must be controlled since childhood. Aim: To assess the association of carotid intima media thickness (CIMT) with the components of the metabolic syndrome in Children. Material and Methods: Cross sectional assessment of 299 children aged $11.5 \pm 0.9$ years ( $58 \%$ women) with and without metabolic syndrome components. Anthropometric parameters and blood pressure were measured and a blood sample was obtained to measure blood glucose and lipids. CIMT was measured using high resolution ultrasound. Results: Ninety three percent of children were post puberal, $64 \%$ were overweight and $25 \%$ had metabolic syndrome. Mean and maximum CIMT correlated with systolic blood pressure $(r=0.21$ and 0.21 respectively $p<0.01)$. Children with a CIMT over the $75^{\text {th }}$ percentile had higher blood pressure and lower HDL cholesterol. A stepwise logistic regression accepted both variables as predictors of CIMT with odds ratios for mean CIMT of 1.46 (1.19-1-79) and 0.81 (0.7-0.94) per five units of change, respectively. Conclusions: In this group of children systolic blood pressure and HDL cholesterol are associated to CIMT.

(Rev Med Chile 2012; 140: 1268-1275).

Key words: Blood pressure; Carotid Intima-Media thickness; Child; Cholesterol HDL.

$\tau$ a prevalencia de las enfermedades crónicas no trasmisibles aumenta en el mundo debido principalmente a la transición nutricional. El sobrepeso y la obesidad constituyen el principal problema y están vinculados, incluso en la edad pediátrica, con síndrome metabólico (SM) y desarrollo de ateroesclerosis subclínica ${ }^{1,2}$.

La prevalencia de obesidad en Chile según la encuesta de salud 2009-2010 comparada con la del 2003 tuvo un incrementó de $61 \%$ a 64,5\% en la población mayor de 15 años $^{3,4}$. La prevalencia nacional de obesidad en menores de 6 años es
$9,7 \%$, mientras que en los escolares de primer año de básico (6-8 años de edad) aumenta a 23,1\% ${ }^{5,6}$.

La enfermedad cardiovascular (ECV) constituye la primera causa de muerte a nivel mundial ${ }^{2}$. En Chile ocupa también el primer lugar en adultos con $25,8 \%{ }^{1}$.

La obesidad en la niñez tiene un amplio rango de complicaciones serias y aumenta el riesgo de enfermedad cardiovascular temprana y muerte precoz en la edad adulta ${ }^{7,8}$. Contribuye desde la niñez a una mayor prevalencia de factores de riesgo cardiovascular del adulto, tales como hiper- 
tensión, dislipidemia y alteración del metabolismo de la glucosa ${ }^{9,10}$. Se propone que la exposición a estos factores de riesgo cardiovascular desde la edad temprana induce cambios en las arterias que contribuyen al desarrollo de la ateroesclerosis en la edad adulta joven ${ }^{11,12}$.

Junto a la obesidad central, representada por la circunferencia de cintura, esos factores constituyen el llamado SM que en adultos predispone al desarrollo de ECV y diabetes mellitus tipo 2 (DM2).

Para los niños no existe consenso para diagnosticar SM y existen varias definiciones, siendo el criterio de Cook S. et al uno de los más utiliza$\operatorname{dos}^{13}$. Los componentes del SM han aumentado también en población pediátrica, con presentación precoz del SM ${ }^{14}$.

La medición del grosor íntimo-medial carotideo, en inglés "carotid intima-media-thickness (CIMT)", a través del ultrasonido de alta resolución, ha demostrado ser un excelente método no invasivo, seguro y capaz de detectar cambios estructurales como engrosamiento a nivel de las arterias carótidas y predecir futuras enfermedades coronarias e infarto de miocardio en la adultez temprana ${ }^{15}$. Una revisión reciente de la literatura señala que en niños o adolescentes con factores de riesgo cardiovascular marcados, como hipertensión arterial y dislipidemia severa, o con diabetes mellitus tipo 1, presentan aumento del $\mathrm{CIMT}^{16}$.

En Chile existen pocos estudios de medición del CIMT tanto en niños como en adultos. En el año 2009 se describió que dos o más componentes del SM presentaban una tendencia no significativa a mayor $\mathrm{CIMT}^{17}$.

El objetivo del presente estudio fue determinar la asociación de los componentes del SM con el aumento del CIMT en una muestra seleccionada de niños y adolescentes escolares chilenos.

\section{Material y Método}

La muestra de 299 niños para este estudio provino de una población de 2.174 escolares de quinto y sexto básico, estudiada transversalmente durante 2009 y 2010 en 14 de las 20 escuelas municipalizadas de la comuna de Puente Alto, participantes del proyecto Fondo Nacional de Desarrollo Científico y Tecnológico (FONDECYT) regular No1090594. Se buscó la presencia de cada uno de los cinco componentes del SM, comparándolos con un subgrupo sin la presencia de los mismos o grupo control. La prevalencia de obesidad y SM en los 2.174 escolares fue de 14,7\% y $7,6 \%$, respectivamente.

En el grupo inicial, una enfermera universitaria y una nutricionista realizaron la evaluación de los niños en cada escuela en horario matinal y se verificó el estado de salud de la semana precedente. Se realizó una encuesta respecto de la ausencia de antecedente de trastornos del metabolismo glucídico, dislipidemias, hipertensión, enfermedades metabólicas, infecciones o terapia farmacológica recientes y de enfermedad cardiovascular familiar. Se solicitó ayuno de $12 \mathrm{~h}$ y los padres o sus representantes firmaron un formulario de consentimiento informado y los niños/niñas mayores de 12 años un formulario de asentimiento informado; estas dos firmas fueron solicitadas nuevamente en esta nueva etapa del proyecto. El estudio fue aprobado por las comisiones de ética de la Escuela de Medicina, Pontificia Universidad Católica de Chile, y del proyecto FONDECYT.

Se midió peso y estatura en balanza de palanca con estadiómetro, marca Seca ${ }^{\circledR}$, con el niño descalzo, con ropa liviana, descontado el peso promedio de ésta y promediándose tres mediciones. Se calculó índice de masa corporal $(\mathrm{IMC}=$ Peso en $\mathrm{kg} / \mathrm{Talla}^{2}$ en $\mathrm{m}$ ) expresados en percentiles con referencia a CDC-NCHS $2000^{18}$. Se catalogó el estado nutricional según percentil de IMC de la misma referencia internacional; eutrofia: 5 al 84, sobrepeso: 85 al 94 , obesidad: $\geq 95$ y desnutrición: $<5$. Se midió perímetro de cintura $(\mathrm{PC})$ con cinta métrica inextensible, sobre el borde lateralsuperior del ilion derecho, en línea medio-axilar, al final de una espiración, según norma internacional y se promediaron tres mediciones ${ }^{19}$; se clasificaron como alterados a aquellos con percentil $\geq 90$ de la misma referencia. Se midió presión arterial con un equipo Dynamap Pro 100, Criticon, según norma internacional y se clasificó como alterada de acuerdo a percentil $\geq 90$ de la misma referencia ${ }^{20}$. La maduración sexual se evaluó según estadios de Tanner mediante un auto-reporte voluntario y privado del estado puberal, con observación de fotos estandarizadas de desarrollo mamario en niñas y genital en hombres, ambas más vello púbico ${ }^{21}$.

Se obtuvieron muestras de sangre venosa, para determinación de lípidos (test enzimático colorimétrico) y glicemia (método Gluco-quant, 
Glucosa/ Hexoquinasa, Roche Diagnostics GmbH, Manheim). Para definir SM se utilizó la recomendación de Cook S. et al ${ }^{13}$, la que considera la presencia de al menos tres componentes de los cinco siguientes: obesidad abdominal representada por la $\mathrm{PC} \geq$ percentil $90^{19}$, presión arterial sistólica (PAS) o presión arterial diastólica (PAD) $\geq$ percentil $90^{20}$, colesterol HDL $(\mathrm{CHDL}) \leq 40$ $\mathrm{mg} / \mathrm{dl}$, triglicéridos $(\mathrm{TG}) \geq 110 \mathrm{mg} / \mathrm{dL}$ y glicemia $\geq 100 \mathrm{mg} / \mathrm{dL}$.

Una vez evaluados los 2.174 alumnos, se reevaluaron en el Laboratorio de Cardiología de la Pontificia Universidad Católica de Chile donde se repitieron las mediciones antropométricas y de laboratorio. También se realizó la medición del CIMT con el método de ultrasonografía vascular de alta frecuencia, basado en un consenso y protocolo internacional, con un equipo ultrasonográfico Phillips LII-3 IE33 y con un transductor linear múltiple-frecuencia 3 a $11 \mathrm{MHz}$ : el valor del CIMT medio y máximo registrado para el análisis estadístico, corresponde al promedio de 6 mediciones del CIMT medio y CIMT máximo, con 3 mediciones en cada carótida ${ }^{22}$. La selección del percentil 75 como punto de corte para clasificar tentativamente el CIMT medio y máximo alterado, permitió analizar con regresión logística la posible influencia sobre este de los componentes del SM.

Se seleccionaron 299 niños, de nivel socioeconómico bajo. Con esta cifra se obtiene el tamaño muestral necesario para realizar una regresión logística paso-a-paso con cinco variables relativas a los componentes del SM (PC, CHDL, TG, PAS-PAD y GLI) y tres variables demográficas (sexo, edad y estado nutricional) con la variable dependiente dicotómica que selecciona $25 \%$ de los casos como posiblemente dañados (CIMT $\geq$ percentil 75 medio y máximo ${ }^{23}$. Los niños que tuvieron la presencia individual o combinada de cada uno de los cinco componentes (PC, CHDL, TG, PAS-PAD y GLI), fueron seleccionados al azar desde la población antes indicada, buscando tener una presencia similar de cada uno de ellos. También se seleccionó al azar un grupo control de 20 sujetos para estimar los promedios de la mayoría de los componentes del SM (excepto TG) con un margen de error de $10 \%$ con una confianza estadística de 95\%.

Para describir variables numéricas se usó promedio y desviación estándar o mediana y rango, según la naturaleza de la variable. Las variables categóricas se presentan como número de casos y porcentaje. Se utilizó test $t$ de Student para muestras independientes para comparación de promedios y test $\chi^{2}$ de Pearson para comparación de proporciones. Para identificar variables asociadas en forma conjunta con CIMT medio o máximo $\geq$ percentil 75 , se usaron modelos de regresión logística paso-a-paso. Se reportan también las razones de chances, en inglés odds ratio (OR), y sus intervalos de confianza al 95\% (IC 95\%), para los efectos significativos en los modelos estimados. Para todos los análisis se usó el programa SPSS 17.0 Se aceptó como significativo todo valor $\mathrm{p} \leq 0,05$.

\section{Resultados}

La muestra del estudio estuvo compuesta por 299 niños con edad promedio de 11,5 $\pm 0,9$ años (rango 10-15 años), 58\% mujeres, 93\% púberes, y $64 \%$ con exceso de peso quienes presentaron $31 \%$ de sobrepeso y $33 \%$ obesidad. La proporción de niños obesos fue $37,6 \%$ mientas que en niñas fue de $31 \%$ (p: 0,05); otras categorías de estado nutricional no tuvieron diferencias.

La proporción o frecuencia de cada componente del SM en la muestra estudiada fue: PC: $56,51 \%$, CHDL: 46,49\%, TG: 42,81\%, PAS: $12,37 \%$, y GLI: $1,33 \%$. Con respecto a la distribución de los componentes del SM: 19\% no cumplió con ninguno, $30 \%$ con uno, $26 \%$ con dos, $22 \%$ con tres y $3 \%$ con cuatro. Es decir, 25\% presentaron SM (Figura 1); 29,3\% de las niñas presentaron SM mientras que en los niños llegó a 19,2\% (p: 0,01).

En la Tabla 1 se muestran los promedios de edad y de las características antropométricas de acuerdo a sexo, sin encontrarse diferencias significativas entre niños y niñas. También se presentan los valores promedios de los componentes del SM y del CIMT según sexo. En los niños los valores estuvieron dentro de rangos normales pero significativamente mayores para glicemia y CHDL con respecto a las niñas, mientras que en ellas los TG fueron mayores y ligeramente fuera de rango considerado como normal para la edad.

En la Tabla 2 se presenta una asociación positiva entre percentil de CIMT percentil $\geq 75$ con la agregación de componentes del SM; la mayor proporción de escolares con CIMT medio $\geq$ per- 


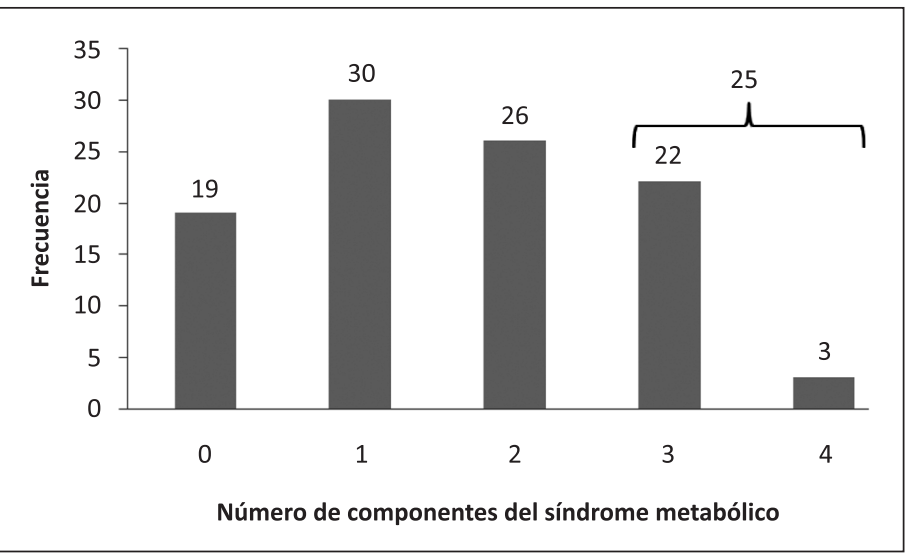

Figura 1. Distribución de los 299 niños según número de componentes del SM.

Tabla 1. Características (promedios $\pm \mathrm{DE}$ ) antropométricas y componentes del SM y del CIMT según sexo

\begin{tabular}{|lcccc|}
\hline Variable & $\begin{array}{c}\text { Total } \\
(\mathbf{n}=\mathbf{2 9 9})\end{array}$ & $\begin{array}{c}\text { Mujeres } \\
(\mathbf{n}=\mathbf{1 7 4})\end{array}$ & $\begin{array}{c}\text { Hombres } \\
(\mathbf{n}=\mathbf{1 2 5})\end{array}$ & $\begin{array}{c}\text { Valor } \\
\mathbf{p}\end{array}$ \\
\hline Edad (años) & $11,5 \pm 1,0$ & $11,5 \pm 0,9$ & $11,6 \pm 1,0$ & Ns \\
\hline Peso (kg) & $53,3 \pm 13,0$ & $54,1 \pm 13,0$ & $52,2 \pm 13,7$ & Ns \\
\hline Talla (cm) & $151,4 \pm 7,0$ & $151,7 \pm 6,6$ & $151,2 \pm 8,3$ & Ns \\
IMC (kg/m²) & $23,0 \pm 5,0$ & $23,3 \pm 4,6$ & $22,6 \pm 4,6$ & Ns \\
z-IMC & $1,1 \pm 1,0$ & $1,1 \pm 0,9$ & $1,06 \pm 1,1$ & Ns \\
\hline PC (cm) & $83,43 \pm 12,5$ & $83,89 \pm 12,0$ & $82,78 \pm 13,3$ & Ns \\
\hline GLI (mg/dl) & $84,10 \pm 7,6$ & $83,20 \pm 6,4$ & $85,36 \pm 8,9$ & 0,01 \\
\hline CHDL (mg/dl) & $43,19 \pm 10,7$ & $41,78 \pm 10,1$ & $45,16 \pm 11,4$ & 0,007 \\
\hline TG (mg/dl) & $113,99 \pm 73,8$ & $124,3 \pm 76,2$ & $99,64 \pm 68,1$ & 0,004 \\
\hline PAS (mmHg) & $111,56 \pm 7,1$ & $111,9 \pm 7,0$ & $111,1 \pm 7,2$ & ns \\
\hline PAD (mmHg) & $55,92 \pm 6,6$ & $55,8 \pm 6,7$ & $56,2 \pm 6,5$ & ns \\
\hline IMTC medio & $0,440 \pm 0,027$ & $0,439 \pm 0,026$ & $0,442 \pm 0,029$ & ns \\
\hline IMTC máximo & $0,451 \pm 0,031$ & $0,450 \pm 0,030$ & $0,452 \pm 0,033$ & ns \\
\hline
\end{tabular}

Tabla 2. Número de componentes de SM según CIMT medio $<0 \geq$ percentil $75(p=0,03)$

\begin{tabular}{|cccccc|}
\hline $\begin{array}{c}\text { Número de } \\
\text { componentes }\end{array}$ & \multicolumn{2}{c}{ Percentil 75 } & \multicolumn{2}{c}{$\geq$ percentil 75 } & Total \\
\hline 0 & 51 & 87,93 & 7 & 12,07 & 58 \\
\hline $1-2$ & 120 & 71,29 & 46 & 27,71 & 166 \\
\hline $3-4$ & 52 & 69,33 & 23 & 30,67 & 75 \\
\hline Total & 223 & & 76 & & 299 \\
\hline
\end{tabular}

centil 75 se encuentra en aquellos con 1-2 y luego 3-4 componentes del SM, destacando que el aumento fue discreto en el grupo de 3 y más componentes (p: 0,03). En el caso de la asociación de CIMT máximo $\geq$ percentil 75 con la agregación de componentes del SM, el resultado fue similar.

Las asociaciones univariadas del CIMT medio y máximo fueron significativas solamente para PAS, con valores de $r$ de Pearson: 0,208 y 0,213 ; sus valores $\mathrm{p}$ fueron 0,0003 y 0,0002 , respectivamente.

Con respecto a la posible asociación de los promedios de los componentes del SM con el aumento de CIMT medio $\geq$ percentil 75 , se observó que tuvieron diferencias estadísticamente significativas solamente para PAS y CHDL (Tabla 3). Una situación similar se presentó para el aumento de CIMT máximo $\geq$ percentil 75 .

El modelo de regresión logística paso-a-paso se ajustó para explicar la variable de respuesta CIMT medio $\geq$ percentil 75 , usando como explicatorias las variables sexo, edad, estado nutricional, PC, CHDL, TG, PAS y PAD (Tabla 4). En este modelo, ingresó en el primer paso la variable PAS y la segunda variable en ingresar fue CHDL. El OR (95\% IC) calculado para el aumento en $5 \mathrm{~mm}$ de $\mathrm{Hg}$ de PAS, controlado por CHDL, fue 1,46 (1,19-1,79). El OR (95\% IC) calculado para la disminución en $5 \mathrm{mg} / \mathrm{dL}$ de CHDL, controlado por PAS, fue $0,81(0,70$ $0,94)$. En el modelo de regresión logística para CIMT máximo $\geq$ percentil 75 como variable de respuesta, los valores de beta estimado y de error estándar fueron similares a la Tabla 4. Las Figuras 2 y 3 muestran la relación de estos dos valores de OR con los componentes del SM seleccionados, ambos para CIMT medio $\geq$ percentil 75 . 
Tabla 3. Relación entre CIMT medio $<0 \geq$ percentil 75 con los componentes del SM (promedios $\pm \mathrm{DE}$ )

\begin{tabular}{|c|c|c|c|}
\hline Variables & $\begin{array}{c}<\text { Percentil } \\
75 \\
(\mathbf{n}=223)\end{array}$ & $\begin{array}{c}\geq \text { Percentil } \\
75 \\
(n=76)\end{array}$ & $\begin{array}{c}\text { Valor } \\
\mathbf{p}\end{array}$ \\
\hline$P C(\mathrm{~cm})$ & $83,02 \pm 12,18$ & $84,60 \pm 13,54$ & ns \\
\hline $\mathrm{GLI}(\mathrm{mg} / \mathrm{dL})$ & $84,29 \pm 6,89$ & $83,55 \pm 9,54$ & ns \\
\hline $\mathrm{CHDL}(\mathrm{mg} / \mathrm{dL})$ & $44,24 \pm 10,86$ & $40,13 \pm 9,80$ & 0,004 \\
\hline TG $(\mathrm{mg} / \mathrm{dL})$ & $111,66 \pm 76,68$ & $120,83 \pm 64,61$ & ns \\
\hline PAS (mmHg) & $110,66 \pm 7,11$ & $114,21 \pm 6,37$ & 0,0001 \\
\hline PAD $(\mathrm{mmHg})$ & $55,63 \pm 6,64$ & $57,01 \pm 6,47$ & ns \\
\hline
\end{tabular}

Tabla 4. Regresión logística paso-a-paso del CIMT medio $\geq$ percentil 75 con las variables que ingresaron al modelo

\begin{tabular}{|lcccr|}
\hline Factor & Estimación & $\begin{array}{c}\text { Error } \\
\text { estándar }\end{array}$ & $\chi^{\mathbf{2}}$ & \multicolumn{1}{c|}{$\begin{array}{c}\text { Valor } \\
\mathbf{p}\end{array}$} \\
\hline PAS & 0,0757 & 0,0206 & 13,586 & $<0,001$ \\
CHDL & $-0,0417$ & 0,0145 & 8,278 & 0,004 \\
\hline
\end{tabular}

\section{Discusión}

En este estudio se analizó la relación que existe entre cada uno de los componentes del SM y el aumento del CIMT en niños con alta prevalencia de sobrepeso y obesidad. En esta muestra el valor promedio fue mayor en las niñas para TG y menor para CHDL con respecto a los niños, hecho que también ha sido comentado por la literatura para este rango etario y se ha observado independientemente del estado nutricional, variando posteriormente con el avance de la pubertad ${ }^{24}$.

La intención de obtener cinco grupos similares en cuanto a número de sujetos, no fue lograda porque muchos casos con el diagnóstico inicial de PAS y de GLI en la primera etapa del estudio, no eran confirmados en el segundo examen, aumentando de esta manera la proporción de casos sin la presencia de algún componente del SM (19\%); este último es el sexto grupo o control que se planificó inicialmente con 20 sujetos y luego llegó a 57 casos. Los segundos exámenes eran realizados en un ambiente más controlado y ello explica que PAS y GLI llegaran sólo a $12,4 \%$ y $1,3 \%$, respectivamente, mientras que hubo

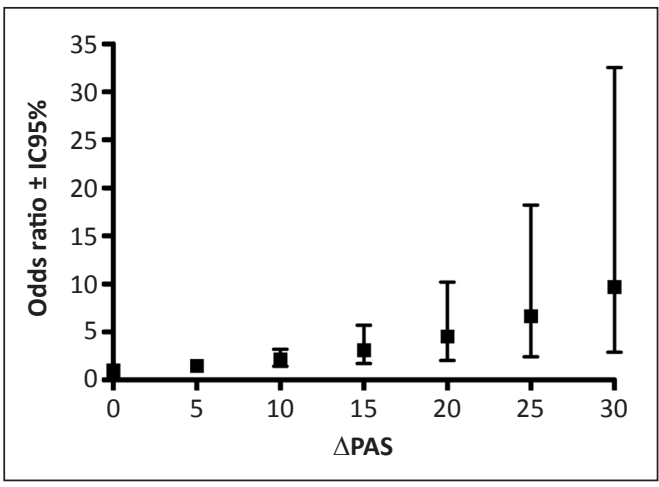

Figura 2. Odds Ratio de CIMT medio $\geq$ percentil 75 , por cada 5 unidades de aumento de PAS ( $\triangle \mathrm{PAS})$, con intervalo de confianza al 95\%.

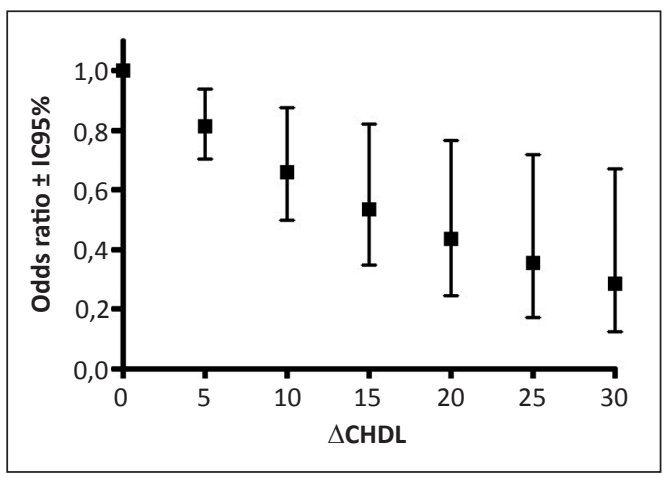

Figura 3. Odds Ratio de CIMT medio $\geq$ percentil 75, por cada 5 unidades de disminución de $C H D L(\triangle C H D L)$, con intervalo de confianza al 95\%.

una relativamente alta proporción de sujetos con presencia de PC, CHDL y TG. Los pocos casos con GLI no tuvieron significación estadística en la mayoría de los análisis.

La sola presencia de uno o dos componentes del SM fue suficiente para mostrar cambios importantes en la proporción de casos con CIMT alterados (Tabla 2). Los promedios de los componentes con CIMT alterado en la tabla 3 también fueron significativos sólo para dos componentes (PAS y CHDL), lo que coincide con los resultados centrales de este estudio, donde la regresión logística, paso a paso, seleccionó los mismos dos componentes del SM con OR significativo.

Nuestro estudio confirma observaciones previas que indican que la ateroesclerosis anátomo-patológica comienza en la infancia 
como enfermedad subclínica ${ }^{25}$. La sumatoria de factores de riesgo cardiovascular, incluyendo el CHDL bajo en la infancia se ha asociado con alteraciones del CIMT $^{14,16,26-29}$.

La obesidad, según IMC, en nuestro estudio no se asoció con incremento de CIMT, a diferencia de otros estudios mencionados en el párrafo anterior. El PC tampoco se asoció aunque se ha demostrado su habilidad diagnóstica para el riesgo cardiovascular en niños y adultos ${ }^{30-32}$. Para ambos casos es posible que con un tamaño muestral mayor se relacionen con CIMT.

Nuestros resultados apuntan a que el CIMT $\geq$ percentil 75 sería un punto de corte adecuado para catalogar como anormal o normal al CIMT en niños. Proponemos a PAS y CHDL como predictores de riesgo de ateroesclerosis subclínica en los niños. Estos hallazgos deben confirmarse con un seguimiento de estos niños para certificar que el daño endotelial, representado por el aumento del CIMT y su relación con la sumatoria de estos predictores, se mantiene o se modifica en el tiempo. La medición con técnicas adecuadas y estandarizadas del CIMT está disponible en la última década y por tanto, no existe una cohorte de niños seguidos por más de ese tiempo con esta técnica ${ }^{33}$. En adultos chilenos se demostró que la PAS también se asociaba significativamente con aumento del CIMT $^{34}$, siendo el presente estudio el primero en demostrarlo en niños.

Este estudio tiene como fortaleza que está basado en una muestra que concentra los factores de riesgo del SM, partiendo de un estudio más amplio cuyos primeros resultados ya han sido publica$\operatorname{dos}^{35,36}$. Este diseño se demostró conveniente para obtener la frecuencia necesaria de componentes del SM, con una muestra total suficiente para poder realizar regresión logística.

Otra de las fortalezas de este estudio es confirmar en niños la importancia de medir la PA, según lo establecido por la práctica clínica pediátrica actual. A pesar de lo anterior, la agencia privada que orienta la práctica clínica en los Estados Unidos de Norteamérica ha señalado que en niños y adolecentes no ha mostrado todavía evidencia suficiente como para recomendar su uso rutinario; sin embargo, la opinión de la misma agencia es recomendar fuertemente su uso en adultos de 18 años y más ${ }^{37}$. Ello sugiere que con nuevos estudios se llegará a la misma recomendación en niños menores de 18 años. Sin embargo, la conclusión de esta agencia privada para estos últimos, no concuerda con las recomendaciones de la Academia Americana de Pediatría, sobre la necesidad imperiosa de medir la presión arterial a todo niño mayor de 3 años para detección oportuna de hipertensión arterial $^{20}$; nuestro grupo ha demostrado que esta recomendación es especialmente válida cuando hay antecedentes familiares de hipertensión arterial $^{38}$. El presente estudio y otros de seguimiento de cohortes, están corroborando la intuición que es indispensable medir la PA y el CHDL a los niños $\mathrm{y}$ adolescentes en los controles de salud ${ }^{28,29}$; esos estudios de cohorte han encontrado asociaciones con el PC aumentado y PAS en el primer caso y con CHDL disminuido en el segundo.

Una posible debilidad en nuestros resultados está referida a que un porcentaje de casos diagnosticados como PAS alterada inicialmente no fueran confirmados en el segundo examen. Ello confirma la necesidad de cumplir las recomendaciones internacionales, que incluyen el reposo necesario y la presencia de un ambiente tranquilo, más la determinación de tres mediciones que luego se promedian ${ }^{20}$. La práctica de ellas mejoró durante el trascurso del estudio original permitiendo que en esta muestra se seleccionaran correctamente los casos con PAS alterada.

\section{Referencias}

1. Mardones F, Velasco N, Rozowski J, et al. Obesidad ¿Qué podemos hacer? Una mirada desde la salud pública. Ediciones Universidad Católica. Santiago, Chile: Salesianos Impresores; 2009.

2. Organización Mundial de la Salud. Diet, nutrition and the prevention of chronic diseases. WHO technical report series 916. Ginebra: OMS; 2003.

3. Ministerio de Salud, Chile [Internet]. Santiago: Encuesta de Salud 2009-2010 [Último acceso: 7 de julio de 2011]. Disponible en: http://www.minsal.gob.cl/portal/ docs/page/minsalcl/g_home/submenu_portada_2011/ ens2010.pdf

4. Ministerio de Salud, Chile [Internet]. Santiago: Resumen ejecutivo Encuesta de Salud 2003, boletín el Vigía no. 20 [Último acceso: 10 de diciembre de 2011]. Disponible en: http://epi.minsal.cl/epi/html/elvigia/VIGIA20.pdf

5. Ministerio de Salud, Chile [Internet]. Departamento de estadísticas (DEIS): Diagnóstico nutricional integrado de la población menor de 6 años, por regiones, diciembre de 2007 (Último acceso: 10 de diciembre de 2011). 
Disponible en: http://163.247.51.54/rem2007/pob dic/12/diag_nutri_1.php?tipomes=12\&tipoano=2007.

6. Junta Nacional de Auxilio Escolar y Becas (JUNAEB) [Internet]. Situación nutricional de los escolares chilenos en establecimientos subvencionados del país. Consultado el 10 de mayo de 2011. Disponible en http://bpt. unaeb.cl:8080/MapaNutricionalGx/

7. Ebbeling CB, Pawlak DB, Ludwig DS. Childhood obesity: public-health crisis, common sense cure. Lancet 2002; 360: 473-82.

8. Freedman DS, Khan LK, Dietz WH, Srinivasan SR, Berenson GS. Relationship of childhood obesity to coronary heart disease risk factors in adulthood: the Bogalusa Heart Study. Pediatrics. 2001; 108: 712-8.

9. Martin BC, Warram JH, Krolewski AS, Bergman RN, Soeldner JS, Kahn CR. Role of glucose and insulin resistance in development of type 2 diabetes mellitus: results of a 25-year follow-up study. Lancet 1992; 340 (8825): 925-9.

10. Burrows R, Bugueño M, Leiva L, Ceballos X, Guillier I, Gattas V, et al. Perfil metabólico de riesgo cardiovascular en niños y adolescentes obesos con menor sensibilidad insulínica. Rev Med Chile 2005; 133 (7): 795-804.

11. Raitakari OT, Juonala M, Kähönen M, Taittonen L, Laitinen T, Mäki-Torko N, et al. Cardiovascular risk factors in childhood and carotid artery intima-media thickness in adulthood. The Cardio-vascular Risk in Young Finns. JAMA. 2003; 290: 2277-83.

12. Juonala M, Magnussen CG, Venn A, Dwyer T, Burns $\mathrm{TL}$, Davis $\mathrm{PH}$, et al. Influence of age on association between childhood and carotid intima-media thikness in adulthood: The Cardiovascular Risk in Young Finns Study, the Childhood Determinants of of Adults Health Study, the Bogalusa Heart Study and the Muscattine Study for the International Childhood Cardiovascular Cohort (i3C) Consortium. Circulation 2010; 122 (24): 2514-20.

13. Cook \$, Weitzman M, Auinger B, Nguyen M, Dietz WH Prevalence of a metabolic syndrome phenotype in adolescents: findings from the third National Health and Nutrition Examination Survey, 1988-1994. Arch Pediatn Adolesc Med. 2003; 157: 821-7.

14. Reinehr T, Wunsch R, Gideon S, Michael A. Relationship between metabolic syndrome definitions for children and adolescents and intima-media thickness. Atherosclerosis 2008; 199: 193-200.

15. Woo KS, Chook P, Yu CW, Sung RY, Qiao M, Leung SS, et al. Overweight in children is associated with arterial endothelial dysfunction and intima-media thickening. Int J Obesity. 2004; 28: 852-7.

16. Kavey R-E W, Simons-Morton DG, de Jesus JM. Expert panel on integrated guidelines for cardiovascular health and risk prediction in children and adolescents: summary report. Pediatrics 2011; 128 (suppl 6): S1-S44.

17. Barja S, Arnaíz P, Acevedo M, Berríos X, Guzmán B, Bambs C, et al. Marcadores de aterosclerosis precoz y Síndrome Metabólico en niños. Rev Med Chile 2009; 137: 522-30.

18. NHANES-2000 CDC Clinical Growth Charts for the United States [Internet]. Disponible en: http://www. cdc.gov/nccdphp/dnpa/growthcharts/training/modules/ module2/text/page6b.htm (Último acceso: 29 de julio de 2011).

19. Fernández J, Redden D, Pietrobelli A, Allison D. Waist circumference percentiles in nationally representative samples of African-American, European American and Mexican American children and adolescents. J Pediatr 2004; 145: 439-44.

20. The Fourth Report on the Diagnosis, Evaluation, and Treatment of High Blood Pressure in Children and Adolescents. National High Blood Pressure Education Program Working Group on High Blood Pressure in Children and Adolescents. Pediatrics 2004; 114: 555-76.

21. Tanner JM, Whitehouse RH. Clinical longitudinal standards for weight, height velocity and stages of puberty. Arch Dis Child. 1976; 51: 170-9.

22. Touboul PJ, Hennerici MG, Meairs S, Adams H, Amarenco $\mathrm{P}$, Bornstein $\mathrm{N}$, et al. Mannheim intima-media thickness consensus (2004-2006). Advisory Board of the 3rd and 4th Watching the Risk Symposium 2004 and 2006, 13th and 15th European Stroke Conference. Cerebrovasc Dis 2007; 23: 75-80.

23. Peduzzi P, Concato J, Kemper E, Holford TR, Feinstein AR. A simulation study of the number of events per variable in logistic regression analysis. J Clin Epidemiol 1996; 49 (12): 1373-79.

24. Magnussen CG, Raitakari OT, Thomson R, Juonala M, Patel DA Viikari JS, et al. Utility of currently recommended pediatric dyslipidemia classifications in predicting dyslipidemia in adulthood: evidence from the Childhood Determinants of Adult Health (CDAH) study, Cardiovascular Risk in Young Finns Study, and Bogalusa Heart Study. Circulation 2008; 117 (1): 32-42.

25. Berenson GS, Srinvasan SR, Bao W. For the Bogalusa Heart Study Association between multiple cardio- vascular risk factors and atherosclerosis in children and young adults. N Engl J Med 1998; 338: 1650-6.

26. Reinehr T, Kiess W, de Sousa G, Stoffel-Wagner B, Wunsch R. Intima media thickness in childhood obesity: relations to inflammatory marker, glucose metabolism, and blood pressure. Metabolism 2006; 55: 113-8.

27. Alagona C, Soro A, Westerbacka J, Ylitalo K, Salonen JT, 
Salonen R, et al. A low high density lipoprotein (HDL) level is associated with carotid artery intima-media thickness in asymptomatic members of low HDL families. Atherosclerosis 2002; 165 (2): 309-16.

28. Toledo-Corral CM, Ventura EE, Hodis HN, Weigensberg MJ, Lane CJ, Li Y, et al. Persistence of the metabolic syndrome and its influence on carotid artery intima media thickness in overweight Latino children. Atheroesclerosis 2009; 206: 594-8.

29. Toledo-Corral CM, Davis JN, Alderete TL, Weigensberg MJ, Ayala CT, Li Y, et al. Subclinical atherosclerosis in Latino youth: progression of carotid intima media thickness and its relationship to cardiometabolic risk factors. J Pediatr 2011; 158: 935-40.

30. Fang J, Zhang JP, Luo CX, Yu XM, Lv LQ, Carotid Intima-media thickness in childhood and adolescent obesity relations to abdominal obesity, high triglyceride level and insulin resistance. Int J Medical Sci 2010; 7 (5): 278-83.

31. Ying Lee CM, Huxley RR, Wildman RP, Woodward M Indices of abdominal obesity are better discriminators of cardiovascular risk factors than BMI: a meta-analysis. J Clin Epidemiol. 2008; 61: 646-53.

32. Freedman DS, Serdula MK, Srinivasan SR Berenson GS. Relation of waist circumferences and skinfold thicknesses to lipid and insulin concentrations in children and adolescents: the Bogalusa Heart Study. Am J Clin Nutr 1999; 69: 308-17.
33. Urbina EM, Williams RV, Alpert BS, Collins RT, Daniels SR, Hayman L, et al. Noninvasive assessment of subclinical atherosclerosis in children and adolescents: recommendations for standard assessment for clinical research: a scientific statement from the American Heart Association. Hypertension 2009; 54 (5): 919-50.

34. Acevedo M, Tagle R, Kramer V, Arnaiz P, Marín A, Pino F, et al. Hipertensión arterial: el factor de riesgo más importante para grosor íntima-media carotídeo elevado y placa carotídea en adultos de Santiago. Rev Med Chile 2011; 139: 290-7.

35. Barja S, Arnaiz P, Domínguez A, Villarroel L, Cassis B, Castillo O, et al. Insulinemia e índice HOMA en niños y adolescentes chilenos. Rev Med Chile 2011; 139: 143543.

36. Mardones F, Villarroel L, Arnaiz P, Barja S, Domínguez A, Castillo O, et al. Prenatal growth and metabolic syndrome components among Chilean children. J of DOHaD, en prensa, 2012.

37. The Guide to Clinical Preventive Service 2007. Recommendations of the U.S. Preventive ServicesTask Force. [Último acceso: 29 de abril de 2012]. Disponible en: https://www.oxhp.com/secure/materials/member, adult_preventive.pdf

38. Aglony M, Arnaiz P, Acevedo M, Barja S, Márquez S, Guzmán B, et al. Perfil de presión arterial e historia familiar de hipertensión en niños escolares sanos de Santiago de Chile. Rev Med Chile 2009; 137: 39-45. 